Pathologe 2021 - 42 (Suppl 2):S188-S189 https://doi.org/10.1007/s00292-021-01021-9 Angenommen: 14. Oktober 2021 Online publiziert: 30 . November 2021

(c) The Author(s), under exclusive licence to Springer Medizin Verlag GmbH, ein Teil von Springer Nature 2021

\section{Bericht aus der wissenschaftlichen Sitzung der AG Informatik}

\author{
Virtuelle Pathologietage der DGP 2021
}

Norman Zerbe

Institut für Pathologie, Charité - Universitätsmedizin Berlin, Berlin, Deutschland

\section{AG-Sitzung}

Auch das Jahr 2021 war weiterhin durch die COVID-19-Pandemie geprägt und erforderte erneut die Durchführung der Jahrestagung als digitale Veranstaltung. Für die AG-Sitzung stand in diesem Jahr ein Zeitraum von $2 \mathrm{~h}$ zur Verfügung.

Eröffnet wurde die Sitzung durch Herrn Prof. Kayser, welcher als Sprecher der AG nach der Begrüßung der Teilnehmer*innen einen Überblicküber das wissenschaftliche Programm gab. In diesem Jahr konnten 2 Gastvorträge von Herrn Prof. Andreas Reiterer aus Freiburg und Herrn Prof. Peter Hufnagl aus Berlin ins Programm aufgenommen werden. Prof. Reiterer gab zunächst einen Überblick über aktuelle Entwicklungen im Bereich der Künstlichen Intelligenz $(\mathrm{KI})$ und präsentierte dabei die Grundlagen unabhängig vom Anwendungsfeld. Anschließend ging er vertiefend auf den Einsatz von $\mathrm{KI}$ in der Makroskopie ein. Prof. Hufnagl stellte in seinem Vortrag die EMPAIA- (Ecosystem for Pathologie Diagnostics with Al Assistance) Initiative vor. Hierbei stellte er zunächst die aktuelle Situation in Deutschland und Europa hinsichtlich der Erstellung für sowie des Einsatzes von KIAlgorithmen in der klinischen Diagnostik dar und fokussierte anschließend auf die Herausforderungen, Ziele sowie den aktuellen Stand des Projekts. Zusätzlich zu diesen Gastrednern wurden 4 Beiträge aus den Einreichungen zur Präsentation und 12 Beiträge als Poster ausgewählt. Bei den Präsentationen, welche im Rah- men der Sitzung der Arbeitsgemeinschaft vorgestellt wurden, handelte es sich um:

- „Digitalization of the workflows in pathology - process analyses as a fundamental approach for a successful roadmap" (PathoScan) von Herrn Prof. Brochhausen (Regensburg),

- „Digital annotations in conventional light microscopy with automatic transfer to whole slide images" von Herrn Bisson (Berlin),

- "Comparison of Sectioning-free Multiphoton Histology to H\&E FFPE Imaging" von Herrn Dr. Strauch (Lübeck),

- „Ultrahigh content imaging for spatial resolution of biobank's archival and in vitro primary resources" von Frau Dr. Bankov (Frankfurt).

Es gab eine aktive Beteiligung der Teilnehmer an den Diskussionen der Beiträge im Anschluss an jeden Vortrag. Fragen konnten hierbei über das Videokonferenzsystem schriftlich gestellt werden. Trotz dieser asynchronen Kommunikation entwickelten sich spannende Diskussionen, welche vertiefende Einblicke in die vorgestellten Arbeiten ermöglichten. Die Diskussionen wurden hierbei durch die AGSprecher Herrn Prof. Kayser und Herrn Zerbe jeweils alternierend moderiert.

\section{Mitgliederversammlung}

Im Anschluss an die wissenschaftliche Sitzung fand die Mitgliederversammlung der Arbeitsgemeinschaft statt. Diese wurde von Herrn Zerbe geleitet, welcher nach der Begrüßung der Teilnehmer und 
der Danksagung für das Engagement der Mitglieder im vergangenen Jahr durch die Tagesordnungspunkte führte. Diese waren: 1) Festlegung der Tagesordnung, 2) Bericht aus der AG, 3) Neuwahlen der AG-Sprecher*innen für die Amtsperiode 2021-2023, 4) Aktivitäten des kommenden Jahres und 5) Verschiedenes.

Nachdem keine weiteren Tagesordnungspunkte durch die Teilnehmer*innen benannt wurden, wurde in Tagesordnungspunkt 2) über die Aktivitäten der Arbeitsgemeinschaft im vergangenen Jahr berichtet. Hier war zunächst die kontinuierliche Zusammenarbeit mit dem Berufsverband und insbesondere mit der Kommission Digitale Pathologie zu nennen. Darüber hinaus wurden in $\mathrm{Zu}$ sammenarbeit mit dem EMPAIA-Projekt im März 20214 halbtägige Weiterbildungskurse organisiert und durchgeführt. Hierbei richteten sich jeweils 2 Kurse an Patholog*innen und KI/IT-Spezialist*innen. Neben grundlegenden Informationen wie Einführung in KI-Technologien sowie der Einführung in die Histologie und Workflows in der Pathologie wurden vertiefend auch regulatorischen und rechtliche Fragenstellungen beleuchtet sowie auch praktische Anwendungsbeispiele aus der Forschung und Routine präsentiert. Als weitere Aktivität aus der AG Informatik, digitale Pathologie und Biobanking ist die Beteiligung am Förderprogram Nationale Forschungsdateninfrastruktur NFDI der DFG zu nennen. Hier haben sich insgesamt 12 Universitätskliniken und Institute, die DGP, der Berufsverband, die TMF und weitere Partner zusammengeschlossen, um unter der Führung der RWTH Aachen mit dem Konsortium NFDI4Patho einen Beitrag für die Pathologie zu leisten.

Im Rahmen des Tagesordnungspunktes 3) wurde entsprechend der Geschäftsordnung eine Neuwahl der AG-Sprecher*innen für den Zeitraum 2021-2023 durchgeführt. Die Amtszeit von Herrn Prof. Kayser, als Sprecher der Arbeitsgemeinschaft, endete entsprechend der Geschäftsordnung nach 4 Jahren. Zur Wahl stellten sich Herr Zerbe, welcher zuvor 2 Jahre als stellvertretender Sprecher der AG tätig war, als neuer Sprecher der AG, sowie Herr Prof. Boor als sein Stellvertreter. Es gab keine weiteren Kandidat*innen. Beide Bewerber wurden durch die an- wesenden Teilnehmer*innen einstimmig für die kommende 2-jährige Amtszeit gewählt.

Anschließend wurden im Tagesordnungspunkt 4) die geplanten Aktivitäten für das kommende Jahr vorgestellt. Hierbei sind insbesondere die Durchführung von weiteren Fortbildungsveranstaltungen für Pathologen und KI/IT-Spezialisten und die damit einhergehende Erhöhung der Sichtbarkeit der Arbeitsgruppe Informatik, Digitale Pathologie und Biobanking i. Allg. sowie der kontinuierlichen Wissensvermittlung im Bereich digitale Pathologie und Anwendung von Methoden der $\mathrm{KI}$ in der Routine im Speziellen zu nennen. Zu diesem Zweck wird die AG weiterhin bei der Ausgestaltung und Durchführung des EMPAIA Academy Formates unterstützen. Ferner soll zusammen mit den nationalen Fachgesellschaften aus Österreich und der Schweiz ein gemeinsames Symposium zur digitalen Pathologie in der DACH-Region organisiert und durchgeführt werden. Es wurde ebenfalls berichtet, dass neben diesen Aktivitäten weiterhin die enge und vertrauensvolle Zusammenarbeit mit dem Berufsverband ein Schwerpunkt der AGTätigkeiten bleiben soll. Im Rahmen der stetigen Prüfung der Geschäftsordnung soll ferner überprüft werden, inwieweit diese im Hinblick auf eine mögliche stärkere Internationalisierung der Zusammenarbeit verfeinert werden sollte.

\section{Fazit}

Zusammenfassend ist festzuhalten, dass trotz oder möglicherweise gerade wegen der COVID-19-Pandemie-Situation und der sich stetig verbessernden technischen Möglichkeiten im Hard- und Softwarebereich die Nutzung digitaler Werkzeuge stetig zunimmt. Auch das Interesse sowie die Beteiligung an entsprechenden Aktivitäten innerhalb und außerhalb der AG steigen wahrnehmbar. Die Nutzung der digitalen Plattformen während der Jahrestagung wurde durch die Mitglieder sehr gut angenommen und es gab eine rege Beteiligung. Wir freuen uns sehr die kommende Jahrestagung 2022 in Münster in Präsenz durchführen zu können und hoffen auf eine weiterhin steigende Teilnehmer*innenzahl.
Korrespondenzadresse

\section{Norman Zerbe}

Institut für Pathologie, Charité - Universitätsmedizin Berlin

Charitéplatz 1, 10117 Berlin, Deutschland norman.zerbe@charite.de

\section{Einhaltung ethischer Richtlinien}

Interessenkonflikt. N. Zerbe gibt an, dass kein Interessenkonflikt besteht.

Für diesen Beitrag wurden von den Autoren keine Studien an Menschen oder Tieren durchgeführt. Für die aufgeführten Studien gelten die jeweils dort angegebenen ethischen Richtlinien.

Funding. The supplement containing this article is not sponsored by industry. 